\title{
Risk assessment for complications of partial nephrectomy - comparative study between retroperitoneoscopic approach and open surgery
}

\author{
Valentin PIRVUT, Nicolae GRIGORE, Adrian HASEGAN, Ionela MIHAI \\ Department of Urology, Faculty of Medicine, "Lucian Blaga" University of Sibiu, Romania
}

\begin{abstract}
- ABSTRACT
Introduction. Partial nephrectomy represent the standard of care for small renal tumor. Laparoscopic approach tends to replace open surgery due to similar oncologic results, but with better aesthetic outcomes and earlier socio-professional reintegration.

Material and methods. Between January 2010 and February 2017, 55 patients ( $p$ ) underwent partial nephrectomy at our center for clinically localized renal tumor, $21 p$ with LPN by retroperitoneal approach and $34 p$ with OPN. All patients had a normal contra-lateral kidney. The selection of patients for nephronsparing surgery was based on preoperative CT scan, location of the tumor, the individual general health status of the patient and individual surgeon preferences.

Results. The mean age of patients with partial nephrectomy was $59.6 \pm 16.4$ years, with $57.9 \pm 11.6$ years for laparoscopic subgroup and $61.3 \pm 12.4$ for open surgery. The transfusion rate was $9.52 \%$ in LPN group, while in the OPN there was no need for transfusion. The warm ischemia time was higher in the LPN group (21.8 7.2 min) than OPN group (15.7 \pm 2.9$)$. The postoperative complication rate is comparable between $L P N$ and OPN (23.81\% v.s. $20.58 \%)$, but the LPN had higher intraoperative complications.

Conclusion. Laparoscopic partial nephrectomy for renal tumors is superior to open surgery by early mobilization of the patient, the low rate of wound complications, short duration of hospitalization, fast socio-professional reintegration of patient, despite the perioperative complication rate that is higher but of low ClavienDindo grade.
\end{abstract}

Keywords: partial nephrectomy, laparoscopic, retroperitoneal, renal tumors, clamping of artery

\section{INTRODUCTION}

Open partial nephrectomy (OPN) has been the gold standard treatment for small renal masses in the first decade of $21^{\text {st }}$ century (1). Laparoscopic partial nephrectomy (LPN) tends to replace OPN due to similar oncologic outcomes, decreased postoperative narcotic use, earlier hospital discharge and a more rapid convalescence (2).

The Preoperative Aspects and Dimensions Used for an Anatomical (P.A.D.U.A.) scoring and the R.E.N.A.L. (radius, exophytic/endophytic properties, nearness of tumor to the collecting system or sinus in millimeters, anterior/posterior location relative to polar lines) scoring were developed in an effort to predict the complications in patients undergoing partial nephrectomy, with an important role on surgical decision-making $(3,4)$.

In the present study we compared renal tumors charactheristics using preoperative renal mass scoring systems (P.A.D.U.A. and R.E.N.A.L. nephrometry) in order to objectify tumor fea- 
tures and identify patients who are at high complications risk.

\section{MATERIAL AND METHODS}

Between January 2010 and February 2017, 55 patients $(p)$ underwent partial nephrectomy at our center for clinically localized renal tumor, $21 \mathrm{p}$ with LPN by retroperitoneal approach and $34 p$ with OPN. All patients had a normal contralateral kidney. The selection of patients for nephron-sparing surgery was based on preoperative CT scan, location of the tumor, the individual general health status of the patient and individual surgeon preferences.

A retrospective chart review was carried out, including age, sex, nephrometry score, operative time (skin opening to skin closing), estimated blood loss (EBL), warm ischemia time (WIT), hospital stay, pathologic stage and surgical margins status.

The perioperative complications were carefully graded using the Clavien-Dindo system.

The work of evaluating classification of complications and calculating nephrometry score was done by 3 individuals.

LPN was performed by a retroperitoneal approach. After positioning the 4 trocars and creating the retroperitoneal work space, the Gerota fascia was opened and the renal artery was isolated. In order to comply with the oncological rules of the procedure the fat surrounding the tumor was excised. The renal artery was then clamped with a vascular bulldog, without clamping the renal vein, and cold excision of the renal mass using round-tipped scissor was performed.

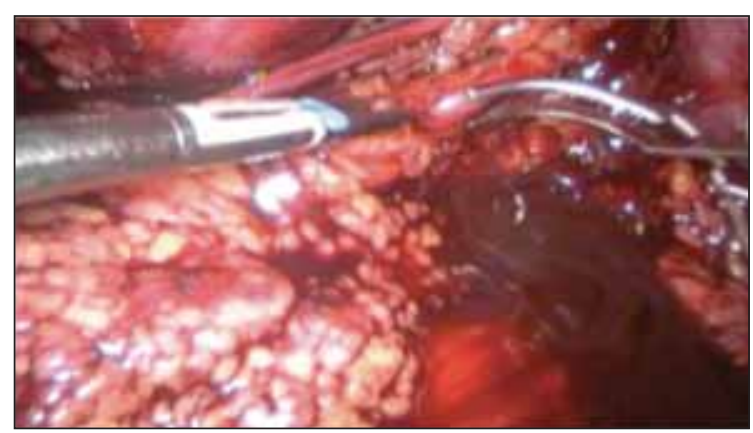

FIGURE 1. Vascular bulldog clamping renal artery

The specimen was placed in a retriving bag, which was then removed at the end of the procedure. Interrupted 3.0 Vicryl suture was perform in order to close the collecting system if it was injured. Interrupted 3.0 Vycril was placed on the renal parenchyma to obtain hemostasis on the segmentary arteries. The renal parenchy- ma defect was closed with 1 Vycril running suture witch was locked at both tail ends with Hem-o-lock clips. After the hemostasis was complete the renal artery was unclamped.

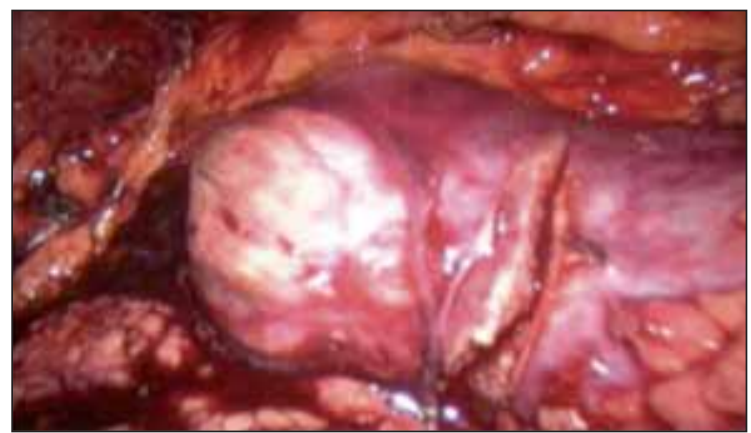

FIGURE 2. Inferior pole renal tumor

For the OPN, afetr clamping of renal artery, the tumor is excised, than the collecting system is sutured with interrupted 3.0 Vycril. The renal parenchyma is closed with 1 Vycril interrupted suture, with or without using hemostatic agents (Gelaspon).

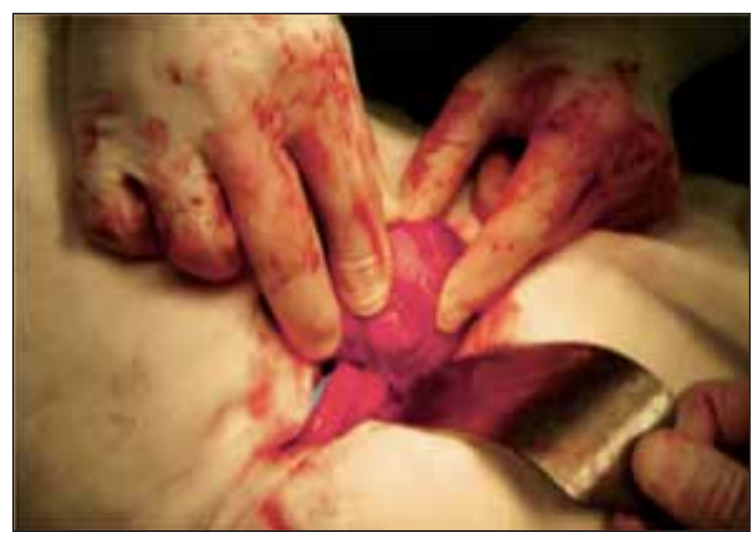

FIGURE 3. Medio-renal tumor

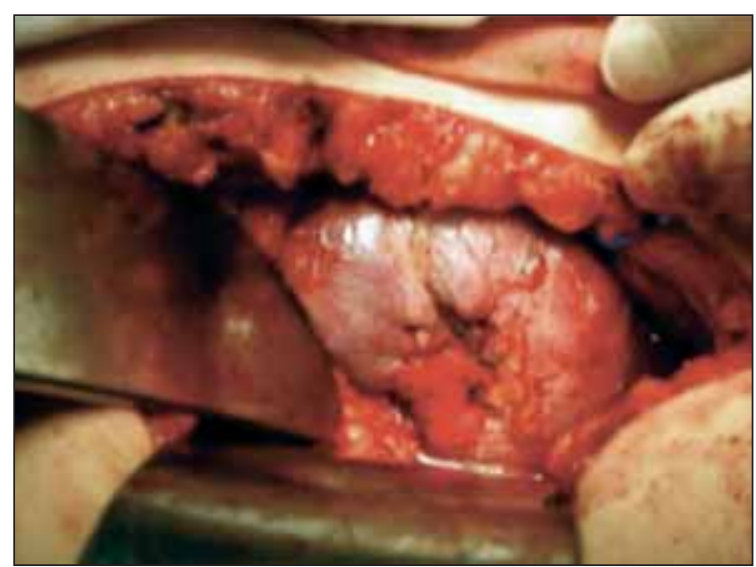

FIGURE 4. Final aspect after renal suturing

The statistical analysis of this paper was done by using chi-square test, the Fischer exact test and the Mann-Whitney $U$ test for paramet- 
ric variables. A p value $<0.05$ was considered statistically significant.

\section{RESULTS}

The mean age of patients with partial nephrectomy was $59.6 \pm 16.4$ years, with $57.9 \pm$ 11.6 years for laparoscopic subgroup and $61.3 \pm$ 12.4 for open surgery. The mean preoperative serum creatinine was the same for both groups of patients. Regarding tumor location the results are comparable between the laparoscopic and open group.

Patients in the OPN had larger tumor size and higher nephrometrie and P.A.D.U.A. scores.

The preoperative comparative characteristics of patients are presented in Table 1.
In our study the mean operative time was longer for LPN group, but with lower blood loss and shorter hospital stay than the OPN group. The transfusion rate was $9.52 \%$ in LPN group, while in the OPN there was no need for transfusion. The warm ischemia time was higher in the LPN group $(21.8 \pm 7.2 \mathrm{~min})$ than OPN group $(15.7 \pm 2.9)$. The percentage of patients requireing collecting system suture in the two groups are approximately the same. The intraoperative and postoperative characteristics are presented in Table 2.

The complications were divided in intraoperative and postoperative subgroups and are presented in Table 3. The postoperative complication rate is comparable between LPN and OPN (23.81\% vs. $20.58 \%$ ), but the LPN had higher intraoperative

TABLE 1

\begin{tabular}{|c|c|c|c|c|}
\hline & All patients & LPN & OPN & p \\
\hline Patients & 55 & 21 & 34 & \\
\hline Mean age (yr) \pm SD (range) & $59.6 \pm 16.4(29-76)$ & $57.9 \pm 11.6(29-72)$ & $61.3 \pm 12.4(32-76)$ & ns \\
\hline $\begin{array}{l}\text { Mean preoperative creatinine (mg/dl) } \\
\pm S D \text { (range) }\end{array}$ & $1.0 \pm 0.4(0.7-1.5)$ & $1.0 \pm 0.4(0.7-1.3)$ & $1.0 \pm 0.4(0.7-1.5)$ & ns \\
\hline \multicolumn{5}{|l|}{ Tumor side } \\
\hline \begin{tabular}{l|l} 
Right \\
\end{tabular} & $18(32.73 \%)$ & $8(38.1 \%)$ & $10(29.41 \%)$ & ns \\
\hline Left & $37(67.27 \%)$ & $13(61.9 \%)$ & $24(70.59 \%)$ & ns \\
\hline \multicolumn{5}{|l|}{ Tumor location } \\
\hline \begin{tabular}{l|l} 
& Upper pole \\
\end{tabular} & $12(21.8 \%)$ & $6(28.6 \%)$ & $6(17.65 \%)$ & ns \\
\hline Mesorenal & $16(29.1 \%)$ & $5(23.8 \%)$ & $11(32.35 \%)$ & ns \\
\hline Lower pole & $27(49.1 \%)$ & $10(47.6)$ & $17(50 \%)$ & ns \\
\hline $\begin{array}{l}\text { Mean CT tumor diameter }(\mathrm{cm}) \pm \mathrm{SD} \\
\text { (range) }\end{array}$ & $3.4 \pm 1.42(1.3-6.2)$ & $3.2 \pm 1.7(1.3-4.9)$ & $3.9 \pm 1.9(1.5-6.2)$ & ns \\
\hline \multicolumn{5}{|l|}{ P.A.D.U.A. score } \\
\hline \begin{tabular}{l|l} 
& Low (6-7) \\
\end{tabular} & $33(60 \%)$ & $15(71.42 \%)$ & $18(52.94 \%)$ & ns \\
\hline Medium (8-9) & $17(30.9 \%)$ & $6(28.58 \%)$ & $11(32.35 \%)$ & ns \\
\hline High (10-14) & $5(19.1 \%)$ & 0 & $5(14.71 \%)$ & ns \\
\hline \multicolumn{5}{|l|}{ R.E.N.A.L. nephrometry } \\
\hline Low (4-6) & $36(65.45 \%)$ & $14(66.66 \%)$ & $22(64.7 \%)$ & ns \\
\hline Medium (7-9) & $15(27.27 \%)$ & $7(33.34 \%)$ & $8(23.53 \%)$ & ns \\
\hline High (10-12) & $4(7.28 \%)$ & 0 & $4(11.77 \%)$ & ns \\
\hline Mean CT tumor volume $(\mathrm{ml}) \pm \mathrm{SD}$ (range) & $37.5 \pm 19.7(16-64)$ & $36.1 \pm 15.4(16-53.4)$ & $40.2 \pm 17.2(19.3-64)$ & ns \\
\hline
\end{tabular}

LPN-laparoscopic partial nephrectomy, OPN-open partial nephrectomy, SD-standard deviation

\section{TABLE 2}

\begin{tabular}{|l|c|c|c|c|}
\hline & All patients & LPN & OPN & p \\
\hline Patients & 55 & 21 & 34 & \\
\hline Operative time (min) \pm SD (range) & $115.7 \pm 42.1(70-230)$ & $154.3 \pm 36.2(90-230)$ & $93.4 \pm 19.8(75-130)$ & 0.025 \\
\hline Blood loss (ml) \pm SD (range) & $259.4 \pm 190.7(100-750)$ & $190.6 \pm 88.6(100-320)$ & $346.7 \pm 155.4(210-750)$ & 0.031 \\
\hline Transfusion rate & $3.63 \%(2 / 55)$ & $9.52 \%(2 / 21)$ & $0 \%$ & $\mathrm{n} / \mathrm{a}$ \\
\hline $\begin{array}{l}\text { Warm ischemia time (min) } \pm \text { SD } \\
\text { (range) }\end{array}$ & $19.4 \pm 9.1(15-39)$ & $21.8 \pm 7.2(19-39)$ & $15.7 \pm 2.9(15-23)$ & 0.024 \\
\hline $\begin{array}{l}\text { Mean postoperative (48 h) serum } \\
\text { creatinine (mg/dl) } \pm \text { SD (range) }\end{array}$ & $1.2 \pm 0.5(0.8-1.8)$ & $1.0 \pm 0.3(0.8-1.4)$ & $1.3 \pm 0.6(0.9-1.8)$ & 0.9 \\
\hline Suture of collecting system & $18(32.72 \%)$ & $7(33.33 \%)$ & $11(32.35 \%)$ & 0.8 \\
\hline Hospital stay & $4.8 \pm 2.6(3-9)$ & $4.1 \pm 1.9(3-7)$ & $5.4 \pm 2.8(4-9)$ & 0.042 \\
\hline
\end{tabular}

LPN-laparoscopic partial nephrectomy, OPN-open partial nephrectomy, SD-standard deviation, n/a - not applicable 
complications. In the LPN group 6 patients presented intraoperative complications, 5 of them developing subcutaneous emphysema.

Postoperative Clostridium difficile infection was similar between the two groups, but with higher urinary tract infection in the OPN group (8.82\% vs. $4.76 \%)$. The complication rate, including urinary fistula, wound infection, bleeding and hematoma are presented in Table 3.

Using the Clavien-Dindo grading system to evaluate OPN complications agaist LPN, in our series Grade 1 rate was 0 vs. 23.81\%, Grade 2 rate was $23.5 \%$ vs. $23.8 \%$ and grade 3 rate was 0 vs $4.7 \%$.

\section{DISCUSSION}

All patients in the study benefit from partial nephrectomy with warm ischemia. The time of warm ischemia was higher in the LPN group (21.8 $\mathrm{min}$ vs. $15.7 \mathrm{~min}$ ) mainly because of the intracorporeal suture which is time consuming.

Renal tumor diameter $(3.2 \mathrm{~cm}$ vs. $3.9 \mathrm{~cm}$ ) and volume (36.1 ml vs. $40.2 \mathrm{ml}$ ) were comparable and had no influence on the outcome of the surgery. When taking into account all characteristics mentioned in the P.A.D.U.A. and R.E.N.A.L. scoring systems, the score obtain had influence on intraoperative and postoperative outcomes, with longer time of ischemia and greater intraoperative blood loss for tumors with medium to high scoring.

In order to have an objectiv comparison of the renal tumors between the two groups we utilized P.A.D.U.A. score and R.E.N.A.L. nephrometry scoring system.

In our series the more complex renal tumors were treated by open surgery because of better intraoperative control and surgeons confort with this approach.
LPN has been shown to be a technically manageable alternative to OPN with similar surgical efficiency. When comparing the complication rate of LPN with OPN the results in the literature are divided, with studies showing higher complications rate in LPN group (6-8) and others in the OPN group $(9,10)$.

In our study the total complication rate is higher in the LPN group with $52.3 \%$ vs. $23.4 \%$, but if we exclude grade I complications, the rate is similar laparoscopic or open surgery.

Urinary fistula was present in the LPN group with a rate of $4.7 \%(1 / 21)$ for a tumor of medium complexity, comparable with high volume studies in the literature that reported urinary leak between 3.1-3.4\% $(6,11)$.

The limitations of our study are its retrospective nature and the lack of long term postoperative evaluation. Another limitation is the reduce number of cases over time, since LPN is practice in our department from January 2014.

\section{CONCLUSIONS}

Laparoscopic partial nephrectomy for renal tumors is superior to open surgery by early mobilization of the patient, the low rate of wound complications, short duration of hospitalization, fast socio-professional reintegration of patient, despite the perioperative complication rate that is higher but of low Clavien-Dindo grade.

P.A.D.U.A. and R.E.N.A.L. nephrometry scoring systems can help the surgeon take the best approach, that can lead to good oncological results with low complications rate.

Acknowledgements: The authors declare no conflict of interests and no sponsorship was provided by the manufacturer of the catheters involved in this study. All authors have read and approved this publication and had equal scientific contribution in publishing this material.

TABLE 3

\begin{tabular}{|l|c|c|c|c|}
\hline Complications & OPN group & LPN group & Overall & p \\
\hline Intraoperative & $1(2.9 \%)$ & $6(28.5 \%)$ & $7(12.9 \%)$ & \\
\hline Subcutaneous emphysema & 0 & $5(23.8 \%)$ & $5(9.0 \%)$ & $\mathrm{n} / \mathrm{a}$ \\
\hline Bleeding requiring transfusion & $1(2.9 \%)$ & $1(4.7 \%)$ & $2(3.7 \%)$ & 0.9 \\
\hline Postoperative & $7(20.5 \%)$ & $5(23.8 \%)$ & $12(21.8 \%)$ & \\
\hline Clostridium difficile infection & $1(2.9 \%)$ & $1(4.7 \%)$ & $2(3.6 \%)$ & 0.8 \\
\hline Wound infection & $2(5.8 \%)$ & 0 & $2(3.6 \%)$ & $\mathrm{n} / \mathrm{a}$ \\
\hline Urinary tract infection & $3(8.8 \%)$ & $1(4.7 \%)$ & $4(7.2 \%)$ & 0.7 \\
\hline Urinary fistula & 0 & $1(4.7 \%)$ & $1(1.8 \%)$ & $\mathrm{n} / \mathrm{a}$ \\
\hline Bleeding requiring transfusion & 0 & $1(4.7 \%)$ & $1(1.8 \%)$ & $\mathrm{n} / \mathrm{a}$ \\
\hline Hematoma & $1(2.9 \%)$ & $1(4.7 \%)$ & $2(3.6 \%)$ & 0.7 \\
\hline Total & $8(23.4 \%)$ & $11(52.3 \%)$ & $19(35.1 \%)$ & \\
\hline
\end{tabular}

LPN-laparoscopic partial nephrectomy, OPN-open partial nephrectomy, n/a - not applicable 


\section{REFERENCES}

1. Ziya Kirkali A. Erdem Canda. Open Partial Nephrectomy in the Management of Small Renal Masses. Advances in Urology Volume 2008, Article ID 309760, 7 pages

2. Gill I.S. et al. Comparative analysis of laparoscopic versus open partial nephrectomy for renal tumors in 200 patients. J Urol. $2003 \mathrm{Jul} ; 170(1): 64-8$.

3. Daniel Canter et al. Utility of the R.E.N.A.L.-Nephrometry Scoring System in Objectifying Treatment Decision-Making of the Enhancing Renal Mass. Urology. 2011 November; 78(5): 1089-1094

4. Darren Desantis et al. The association between renal tumour scoring system components and complications of partial nephrectomy. Can Urol Assoc J 2015; 9(1-2):39-45
5. Rosaleen B. Parsons et al. RENAL Nephrometry Scoring System: The Radiologist's Perspective. AJR 2012; 199:W355-W359

6. Jennifer E. Reifsnyder, Ranjith Ramasamy et al. Laparoscopic and Open Partial Nephrectomy: Complication Comparison Using the Clavien System. JSLS (2012)16:38-44

7. Polascik T.J., Pound C.R., Meng M.V., Partin A.W., Marshall F.F. Partial nephrectomy: technique, complications and pathological findings. J Urol. 1995; 154(4):1312-1318

8. Van Poppel H., Bamelis B., Oyen R., Baert L. Partial nephrectomy for renal cell carcinoma can achieve long-term tumor control. J Urol. 1998; 160(3 Pt 1):674 - 678.
9. Marszalek M., Meixl H., Polajnar M., Rauchenwald M., Jeschke K., Madersbacher S. Laparoscopic and open partial nephrectomy: a matched-pair comparison of 200 patients. Eur Urol. 2009; 55(5):1171-1178.

10. Zini L., Patard J.J., Capitanio U., et al. The use of partial nephrectomy in European tertiary care centers. Eur J Surg Oncol. 2009; 35(6):636 - 642

11. Gil I.S., Kavoussi L.R., Lane B.R. et al. Comparison of 1800 laparoscopic and open partial nephrectomies for single renal tumors. J Urol. 2007; 178(1):41-46 